\title{
Changes in magnetic resonance mammography due to hormone replacement therapy
}

\author{
Stefan OR Pfleiderer, Steffen Sachse, Dieter Sauner, Christiane Marx, Ansgar Malich, \\ Susanne Wurdinger and Werner A Kaiser
}

Institute of Diagnostic and Interventional Radiology, Friedrich-Schiller-University, Jena, Germany

Corresponding author: Stefan OR Pfleiderer (e-mail:stefan.pfleiderer@med.uni-jena.de)

Received: 19 Nov 2003 Revisions requested: 8 Jan 2004 Revisions received: 14 Jan 2004 Accepted: 24 Feb 2004 Published: 16 Mar 2004

Breast Cancer Res 2004, 6:R232-R238 (DOI 10.1186/bcr779)

(C) 2004 Pfleiderer et al., licensee BioMed Central Ltd. This is an Open Access article: verbatim copying and redistribution of this article are permitted in all media for any purpose, provided this notice is preserved along with the article's original URL.

\begin{abstract}
Background: The aim of the present article is to investigate effects of hormone replacement therapy (HRT) on contrast medium enhancement patterns in postmenopausal patients during magnetic resonance mammography (MRM).

Materials and methods: Two hundred and fifteen patients receiving hormonal medication were divided into four groups: 150 patients with 1 MRM during HRT (group A), 13 patients with 2 MRMs under HRT (group B), 30 patients with 1 MRM during HRT and 1 MRM after HRT withdrawal (group C), and 22 women with $1 \mathrm{MRM}$ after HRT withdrawal (group D). Dynamic MRM was performed at 1.5 Tesla. Signal intensity changes were characterized by five time curves: minimal enhancement (type I), weak continuous enhancement (type II), strong continuous enhancement (type III), and a steep initial slope followed by a plateau phenomenon (type IV) or by a washout effect (type V).
\end{abstract}

Results: Of all 193 patients under HRT (group A + group B + group C), 60 patients (31.1\%) showed curve type I, 88 patients (45.6\%) showed type II and 45 patients (23.3\%) showed type III. There were significant differences to 52 patients after HRT withdrawal (group $\mathrm{C}+$ group D) $(P<0.0001)$, with 42 patients $(80.8 \%)$ for curve type I, 8 patients $(15.4 \%)$ for type II, and 2 patients (3.8\%) for type III. In both MRM sessions in group B, $69 \%$ of the patients showed identical curve types without significant differences $(P=0.375)$. In group $C, 28$ of 30 patients $(93 \%)$ dropped to lower curve types with significant differences in curve types during and after HRT $(P<0.0001)$.

Conclusion: The majority of patients receiving postmenopausal HRT showed bilateral symmetrical, continuous enhancement without evidence of a plateau phenomenon or a washout effect due to HRT in MRM. Hormonal effects could be proven and were reproducible and reversible.

\section{Introduction}

Observational studies have shown that women receiving hormone replacement therapy (HRT) have a reduced risk for osteoporosis [1,2] by preventive slowing of the decrease of bone mineral density in postmenopausal women. Protective effects against heart disease have also been claimed $[3,4]$. In contrast, Grimes and Lobo recently reported an increased risk for cardiovascular disease and breast cancer in the Women's Health Initiative trial of HRT [5]. The relation of HRT and the increased risk for breast cancer has been controversially discussed for several years $[6,7]$.
It is well known that mammographic density of the breast is increased during HRT and potentially affects the diagnosis of breast cancer on mammograms $[8,9]$. Increased density may therefore obscure mammographic masses or may decrease the detection rate of microcalcifications, resulting in more false-negative findings in mammography and, consecutively, resulting in a decrease in the sensitivity of mammographic screening [10]. Even the specificity of mammography seems to be reduced with postmenopausal HRT [11]. There are differences between women using combination HRT compared with women treated with estrogens alone [12]. 
Table 1

\begin{tabular}{|c|c|c|c|}
\hline Group & $\begin{array}{c}\text { Examinations during } \\
\text { HRT }\end{array}$ & $\begin{array}{l}\text { Examinations after } \\
\text { HRT withdrawal }\end{array}$ & Data evaluation \\
\hline Group A $(n=150)$ & 1 & & \\
\hline Group B $(n=13)$ & 2 & & Test for reproducibility \\
\hline Group C $(n=30)$ & 1 & 1 & Test for effect of HRT (dependent samples) \\
\hline Group D $(n=22)$ & & 1 & \\
\hline $\begin{array}{l}\text { Group A + group B, } \\
\text { first examination }+ \text { group C, } \\
\text { first examination }(n=193)\end{array}$ & 1 & & Distribution of curve types under HRT \\
\hline $\begin{array}{l}\text { Group } C \text {, second examination + } \\
\text { group } D(n=52)\end{array}$ & & 1 & Distribution of curve types after HRT withdrawal \\
\hline Total $(n=215)$ & & & \\
\hline
\end{tabular}

Two hundred and fifteen patients were divided into four groups related to the time relationship between HRT and magnetic resonance mammography and the numbers of examinations. Examinations after HRT withdrawal were performed 1-3 months after termination.

The ultrasound examination of the breast is less influenced by HRT. It has been reported that formation of cysts detectable by ultrasound does not significantly correlate with HRT [13]. In women with breast tumors receiving HRT, an increase in the number of intratumoral vessels and an alteration of the resistance and pulsatile index were associated with the menopausal status [14].

Several reports have described hormonal influences and alterations of the contrast enhancement kinetics in dynamic magnetic resonance imaging (MRI) of the breast. Alterations in T1 and T2 relaxation times depending on the phase of the menstrual cycle were reported in 1985 [15]. In contradiction, Martin and El Yousef did not observe differences of the T2 relaxation time in different cycle phases [16]. Regarding the contrast enhancement patterns in magnetic resonance mammography (MRM), it has been reported that during the second half of the menstrual cycle a generally higher contrast medium (CM) uptake of fibrocystic formations [17], and even of normal breast parenchyma [18], takes place. Diffuse or focal enhancement may occur in postmenopausal women who are taking HRT [19]. Other authors report volume changes of the breast or alterations of the tissue component such as the ratio between fatty tissue and breast parenchyma, the water content, and the fibroglandular fraction during the menstrual cycle $[20,21]$.

The purpose of the present study was to evaluate the effects of HRT on contrast-enhancement kinetics in MRM in postmenopausal women.

\section{Materials and methods}

Between December 1994 and April 1999, 1420 patients who had lesions of uncertain dignity in X-ray mammo- graphy or ultrasound of the breast underwent MRM at our institution. The patients gave their written consent prior to the examination. Two hundred and fifteen postmenopausal women among these patients (mean age, $52 \pm 12.2$ years) were treated with HRT. They were retrospectively divided into four groups (groups A-D) according to the time of onset or withdrawal of HRT and of MRM: 150 patients received 1 MRM during HRT (group A), 13 patients underwent 2 MRMs under HRT (group B), 30 patients received 1 MRM during HRT and 1 MRM within 1-3 months after HRT withdrawal (group C), and 22 women received 1 MRM within 1-3 months after withdrawal of hormonal medication (group D) (Table 1).

All MRM studies were performed on a 1.5 Tesla magnetic resonance scanner (ACS II; Philips, Best, The Netherlands). Patients lay in a prone position on a dedicated double breast coil. Subsequent to acquisition of scout images in three orthogonal orientations, a T1-weighted twodimensional gradient echo sequence (repetition time/echo time/flip angle = 12/4.1/30; thickness, $4 \mathrm{~mm}$; field of view, $350 \times 350 \mathrm{~mm}^{2}$; matrix, $256 \times 256$; seven slices; acquisition time, $1 \mathrm{~min}$ and $25 \mathrm{~s}$ ) in the coronal orientation and a T2-weighted turbo spin echo sequence (repetition time/echo time/flip angle $=14286 / 300 / 90$; turbo factor, 29; slice thickness, $4 \mathrm{~mm}$; gap, $0.4 \mathrm{~mm}$; field of view, $350 \times 350 \mathrm{~mm}^{2}$; matrix, $256 \times 256$; 24 slices; acquisition time, $3 \mathrm{~min}$ and $5 \mathrm{~s}$ ) in the transverse orientation were acquired. Dynamic contrast-enhanced scans were performed using a multislice, T1-weighted, twodimensional gradient echo sequence in the transverse orientation (repetition time/echo time/flip angle =96/5/80; slice thickness, $4 \mathrm{~mm}$; gap, $0.4 \mathrm{~mm}$; field of view, $350 \times 350 \mathrm{~mm}^{2}$; matrix, $256 \times 256$; 24 slices; acquisition time, $1 \mathrm{~min}$ ) at 1 -min intervals before and after intravenous 
administration of a bolus of $0.1 \mathrm{mmol} / \mathrm{kg}$ dosage of $\mathrm{Gd}$ DTPA (Magnevist; Schering, Berlin, Germany) for $8 \mathrm{~min}$.

For semiquantative analysis of the dynamic study, timesignal intensity (SI) curves were calculated and subtraction of the precontrast images from the postcontrast images was performed using the built-in software of the magnetic resonance scanner. Regions of interest were placed in each area or spot that showed CM enhancement. Five curve types concerning the amount of $\mathrm{CM}$ enhancement were predefined according to the report of Kaiser and Zeitler [22]. The curves were characterized by minimal enhancement (up to 25\% increase of the SI after administration of Gd-DTPA; curve type I), weak continuous enhancement (increase of SI between 25\% and 60\%; curve type II), strong continuous enhancement (increase of $\mathrm{SI}$ between $60 \%$ and $80 \%$; curve type III), and a steep initial slope (more than $80 \%$ increase of $\mathrm{SI}$ ) followed by a plateau phenomenon (curve type IV) or by a washout effect (curve type V) (Fig. 1). Curve type IV is suspicious and curve type $\mathrm{V}$ is highly suspicious for a malignant tumor in MRM. Curve types I-III are more typical for benign lesions [22]. In cases where several areas or spots with different curve types were present in the same breast, the curve type most suspicious for malignancy was chosen for evaluation.

The Kruskal-Wallis test for single-ordered row $\mathrm{x}$ column tables was used to evaluate statistical differences between the incidence of the five curve types of all patients examined during HRT (group A, group B and group C) and those patients who underwent MRM without hormonal medication (group C and group D). This test was also used for testing whether group $D$, which was examined without HRT, differed notably in its SI curve distribution compared with all other patients receiving HRT (group A, group B and group C) and whether group $D$ would thus be representative for that sample. For statistical evaluation of differences between the curve types achieved in the two examinations during and after withdrawal of HRT in group C, an exact Wilcoxon signed rank test for dependent samples was performed. The same test was used to evaluate differences between the two measurements during HRT in group B.

\section{Results}

Of all patients receiving HRT at the time of examination (group $\mathrm{A}$; group $\mathrm{B}$, first examination; group $\mathrm{C}$, first examination), 60 of 193 patients (31.1\%) showed curve type I, 88 patients (45.6\%) were referred to as curve type II, and 45 patients (23.3\%) showed curve type III (Fig. 2). $\mathrm{CM}$ enhancement characteristics were homogeneous or spotted granular, were continuous over the time of dynamic imaging and were symmetrical in both breasts. One of the patients with curve type II had an additional segmental
Figure 1

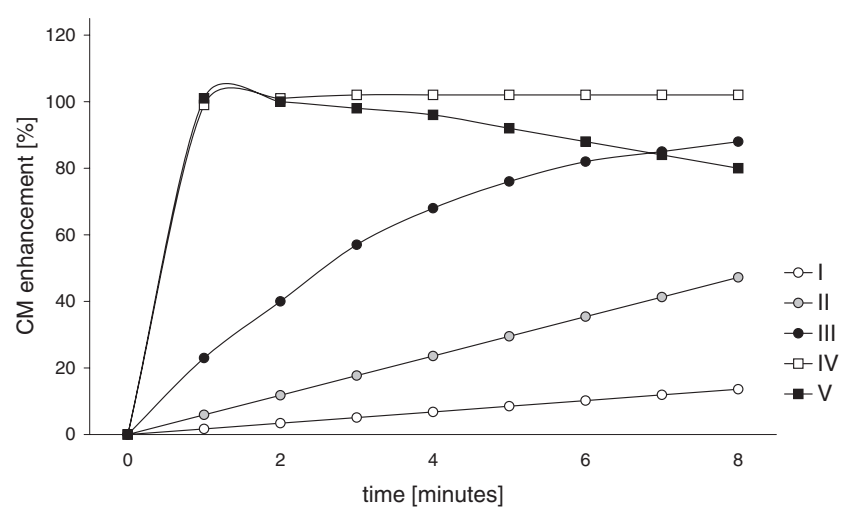

Five predefined curve types I-V dependent on contrast medium (CM) enhancement behavior. Curve type I showed a minimal and continuous increase of postcontrast signal intensity $(\mathrm{SI})$ up to $25 \%$. Curve type II described a weak continuous increase of postcontrast SI by $25-60 \%$. A strong enhancement after the injection of $\mathrm{CM}$ with a continuous increase between $60 \%$ and $80 \%$ is demonstrated by curve type III. Curve type IV and curve type $\mathrm{V}$ represent a very strong increase with a steep slope of the SI of more than $80 \%$ during the first 2 min after CM administration, followed by a plateau phenomenon with curve type IV and followed by a washout effect with curve type $\mathrm{V}$.

type IV in the right breast. The histological evaluation after surgical excision revealed a ductal carcinoma in situ in the right breast. One of the patients with curve type III revealed a breast lesion on the right side, which presented $\mathrm{CM}$ enhancement according to curve type $\mathrm{V}$ with an increase of $112 \%$ of SI after CM administration suspicious of breast cancer. Histological evaluation after surgery revealed an invasive lobular cancer.

Patients who received MRM under HRT $(n=193)$ showed highly significant differences in the contribution of the curve types compared with the patients who were free of hormone medication at the time of MRM (group $D$ and group $\mathrm{C}$ after withdrawal of HRT) $(P<0.0001)$. Forty-two of these 52 patients (80.8\%) showed curve type I, 8 patients (15.4\%) showed curve type II, and 2 patients (3.8\%) were referred to as curve type III (Fig. 2).

The group of 13 patients who received two MRM examinations during HRT (group B) showed hormonal effects with comparable signal intensities in both MRI examinations. Eight patients showed identical curve types in examination 1 and examination 2: six of them showed curve type II, one of them showed curve type I and one of them showed curve type III. Three patients were referred to as curve type II in the first examination and changed to curve type III in the second MRM session, whereas one patient changed from curve type III to curve type II and one patient changed from curve type I to curve type II. Statistical analysis using the Wilcoxon signed rank test 
Figure 2

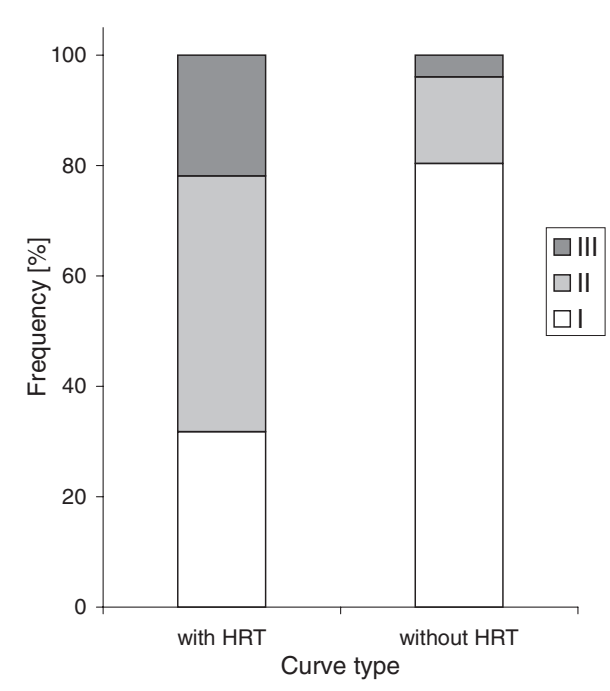

The contribution of the curve types of patients under hormone replacement therapy (HRT) (group A; group B, first examination; group $C$, first examination; $n=193)$. Sixty patients $(31.1 \%)$ showed curve type I, 88 patients $(45.6 \%)$ showed curve type II and 45 patients $(23.3 \%)$ showed curve type III. These findings were statistically highly significant $(P<0.0001)$ compared with patients without hormonal medication (group $\mathrm{C}$, second examination; group $\mathrm{D} ; n=52$ ). In the group free of hormonal medication, 42 patients $(80.8 \%)$ showed curve type I, 8 patients (15.4\%) showed curve type II and 2 patients (3.8\%) showed curve type III.

revealed no significant differences between the two examinations under HRT $(P=0.375)$.

Twenty-eight of 30 patients of group $\mathrm{C}$ changed to lower curve types in the second MRM session after withdrawal of HRT. Twelve patients dropped by two curve types after withdrawal of HRT (Figs 3 and 4). Sixteen patients dropped by one curve type after stopping HRT and two patients remained at curve type II. The Wilcoxon signed rank test revealed that the curve types under HRT are highly significant different compared with the curve types after withdrawal of hormone medication $(P<0.0001)$.

\section{Discussion}

The current study demonstrates bilateral, symmetrical, synchronous, and progressive $\mathrm{CM}$ enhancement in the breast in dynamic MRM according to the findings of the majority of the patients receiving HRT. Classifying these patients in time-SI curve types revealed enhancement patterns according to curve types I, II, and III that are not suspicious of malignancy.

The statistically significant difference in curve types with and without HRT underlines the presence of hormonal effects in MRM. Neither a plateau phenomenon nor a washout effect, which are known to be typical for invasive breast cancer [23], occurred during HRT. In our study
Figure 3
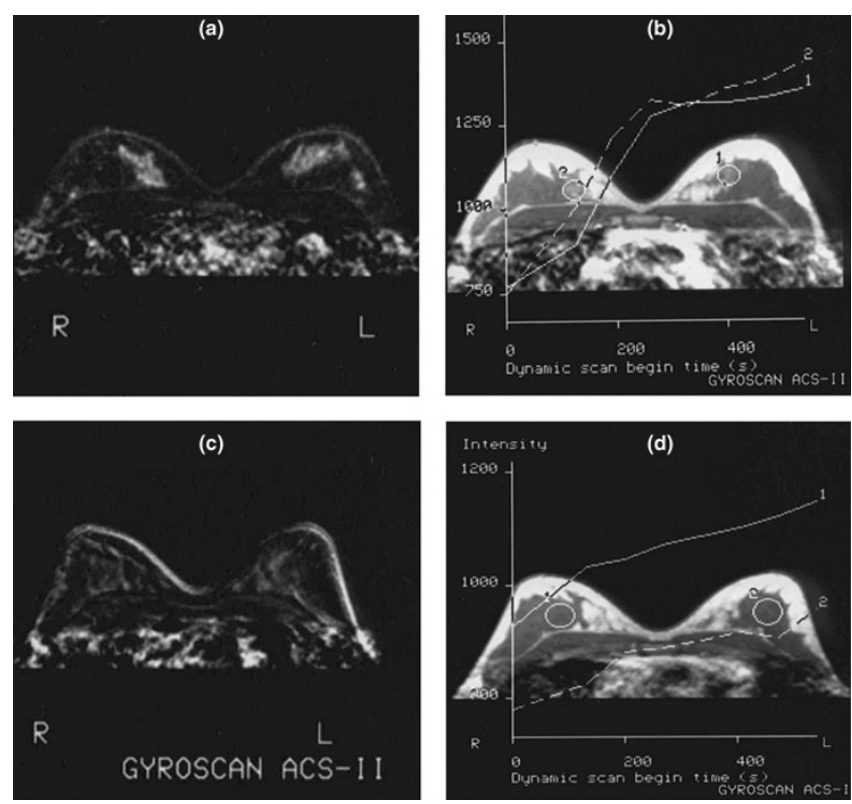

Magnetic resonance mammography of a 47-year-old patient during hormone replacement therapy (HRT) and after withdrawal of hormonal medication. (a) The T1-weighted subtraction image $180 \mathrm{~s}$ after contrast medium (CM) administration showed a bilateral symmetrical continuous enhancement without a plateau phenomenon or a washout effect of both breasts. (b) The signal intensity increased by about 75\%. The CM enhancement was therefore curve type III. (c) A second magnetic resonance examination after stopping HRT revealed almost no visible $\mathrm{CM}$ enhancement. (d) Compared with the first magnetic resonance session during HRT, the time-intensity curve dropped from type III to type I. R, right; L, left.

HRT therefore did not result in mimicking breast cancer typical findings in dynamic MRM. Carcinomas in situ (CIS), on the contrary, might present with similar findings to hormonal effects in MRM and therefore may complicate differential diagnosis. CIS very often have unilateral, focal formed, local limited, and multifocal signal enhancements. In cases of bilateral CIS, which is reported to be up to $40 \%$ in lobular CIS [24], differentiation may be uncertain. The detection of CIS may thus be obscured, especially with low-grade CIS, because they may present with patchy continuous enhancement just like parenchymal CM enhancement under HRT in the present study [25]. Stopping HRT prior to a second MRM is thus advisable.

The limitations of the current study are as follows. The study design was retrospective. The size of the four patient groups with and without hormonal medication was therefore different. However, the particular findings of contrast enhancement were proven statistically significant. Additionally, the type of hormone medication is rather heterogeneous (e.g. combination therapy versus monotherapy or oral application versus transdermal application). 
Figure 4
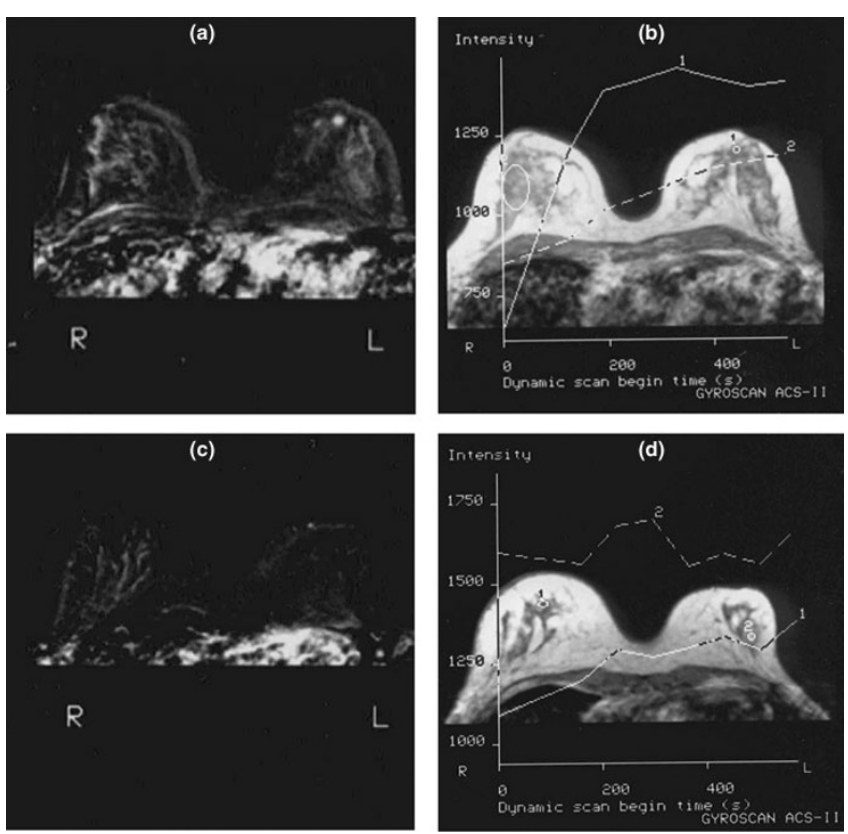

Magnetic resonance mammography (MRM) of a 53-year-old patient during hormone replacement therapy (HRT) with a small histologically proven invasive lobular cancer in the left breast. (a) The subtraction image $420 \mathrm{~s}$ after contrast medium (CM) administration showed a bilateral, almost symmetrical, enhancement. (b) The time-intensity curve of the right breast indicated a continuous rise of the signal intensity of $41 \%$ (curve type II). Additionally, a round blurred area of stronger CM enhancement was detectable just behind the nipple of the left breast. The lesion was not obscured by hormone-associated $\mathrm{CM}$ enhancement and showed a rapid CM enhancement followed by a plateau phenomenon, which is characteristic for curve type IV suggesting suspicion of malignancy. Eight months after breastconserving therapy, a MRM for follow-up was performed without hormonal medication. (c) The subtraction images showed a weaker bilateral enhancement. (d) The time-intensity curve indicated a weak increase of signal intensity less than $25 \%$, reflecting a decrease by one curve type after withdrawal of HRT. R, right; L, left.

Reichenbach and colleagues found an elevated parenchyma/fat tissue ratio in women who were administered combined hormonal therapy [21]. Growing volumes of breast parenchyma might be an explanation for the increase of $\mathrm{CM}$ enhancement because breast parenchyma has a higher microvessel density than fatty tissue, contributing to extravascular CM passage into the interstitial space. CM enhancement in malignant tumors may also be related to an increased fractional volume of the extravascular extracellular space [26].

Studies of breast cell proliferative activity showed that most proliferation, as well as the highest breast density on mammograms, occurs in the luteal phase of the menstrual cycle, which is partially characterized by high serum levels of progesterone and estrogens [27]. Breast density is also breast parenchyma probably results in increased contrast enhancement during dynamic contrast-enhanced MRI.

Several reports deal with the menstrual cycle and its effects on relaxation times, breast tissue composition, and CM enhancement in MRM [15-19]. Graham and colleagues [23] and Fowler and colleagues [29] found increased water content and rising fibroglandular tissue volume of the breast during the end of the menstrual cycle and the menses. Rieber and colleagues reported an increase of $\mathrm{CM}$ enhancement during the second half of the menstrual cycle [17]. In this secretory cycle phase, the levels of estrogene and progesterone are physiologically elevated. This situation could thus be interpreted as an endogenous hormonal stimulation, and it seems to have similar effects on dynamic MRM compared with exogenous hormone administration. On the contrary, the higher CM enhancement during HRT may obscure overlaying pathologies. However, two cases with histologically proven cancers demonstrated that the detection of an invasive cancer or a ductal carcinoma in situ was not obscured inevitably in these cases.

Kuhl and colleagues found that 26 of 60 enhancing foci demonstrated enhancement velocity beyond the malignancy threshold of more than $80 \%$ increase of the signal intensity after the first minute after contrast media administration [30]. But these cases did not show any washout effect, which in our opinion is the strongest indicator for malignancy, and a plateau phenomenon was present in only two out of 60 lesions. Taking this into account, the study results are comparable and similar. However, the few differences between our findings and other reports may be due to different imaging protocols because MRM still is not standardized.

Hormonal effects are well known in X-ray mammography as well and do not similarly affect all women. Breast density on mammograms during HRT increases in only $20-35 \%$ of postmenopausal women $[10,11]$. In contrast to MRM, increased breast density in patients under HRT may even cause a decrease of sensitivity of breast cancer detection in screening mammography $[12,13]$.

The statistically nonsignificant differences between the first and the second examination in group $B$ reflect the equivalence of the two MRM examinations during HRT. The differences also underline the reproducibility of enhancement patterns during HRT.

The statistically highly significant differences in group C indicate that alterations of $\mathrm{CM}$ enhancement patterns caused by HRT are reversible after stopping hormonal medication, comparable with the cyclic reversible changes in MRM during the secretory phase of the menstrual cycle [23]. 
Two patients showed higher curve types (curve type III) during HRT than during treatment with selected estrogene receptor modulators. These findings again underline that hormone effects are reversible under selected estrogene receptor modulator therapy as well, but the patient groups are too small for statistically proven comparison of hormone withdrawal and following treatment using selected estrogene receptor modulators. Bogin and Degani, in contrast, recently reported higher levels of vascular endothelial growth factor after treatment with tamoxifen compared with HRT resulting in higher tumor vascular permeability, leading to increased $\mathrm{CM}$ enhancement of MCF7 breast cancers. Tumor vessels seem to behave in a different manner to vessels of normal breast tissue, which may be due to the pathological high leakage of tumor vessels [31].

\section{Conclusion}

The majority of postmenopausal patients receiving HRT resulted in characteristic changes with bilateral, symmetrical, and continuous CM enhancement in dynamic MRM. Cancer-typical findings like a plateau phenomenon or a washout effect could not be observed, and in two examples even breast cancer diagnosis was not affected. Repeated examinations under HRT resulted in reproducible hormone effects in MRM, which were reversible after termination of HRT. In cases of uncertain suspicious findings, which may be rarely present during HRT, MRM could thus be repeated sufficiently after hormone withdrawal.

\section{Competing interests}

None declared.

\section{References}

1. Gibaldi M: Prevention and treatment of osteoporosis: does the future belong to hormone replacement therapy? J Clin Pharmacol 1997, 37:1087-1099.

2. Lindsay R: Hormone replacement therapy for prevention and treatment of osteoporosis. Am J Med 1993, 95:37S-39S.

3. Stampfer M, Colditz GA, Willett WC, Manson JE, Rosner B, Speizer FE, Hennekens $\mathrm{CH}$ : Postmenopausal estrogen therapy and cardiovascular disease. Ten-year follow-up from the Nurse's Health Study. N Engl J Med 1991, 325:1758-1766.

4. Rich-Edwards JW, Manson JE, Hennekens $\mathrm{CH}$, Buring JE: The primary prevention of coronary heart disease in women. Engl J Med 1995, 332:1758-1766.

5. Grimes DA, Lobo RA: Perspectives on the Women's Health Initiative trial of hormonal replacement therapy. Obstet Gynecol 2002, 100:1344-1353.

6. Daling JR, Malone KE, Doody DR, Voigt LF, Bernstein L, Coates RJ, Marchbanks PA, Norman SA, Weiss LK, Ursin G, Berlin JA, Burkman RT, Deapen D, Folger SG, McDonald JA, Simon MS, Strom BL, Wingo PA, Spirtas R: Relation of regimens of combined hormone replacement therapy to lobular, ductal, and other histologic types of breast carcinoma. Cancer 2002, 95: 2455-2465.

7. Persson I, Thurfjell E, Bergstrom R, Holmberg L: Hormone replacement therapy and the risk of breast cancer. Nested case-control study in a cohort of Swedish women attending mammography screening. Int J Cancer 1997, 72:758-761.

8. Harvey JA, Pinkerton JV, Herman CR: Short-term cessation of hormone replacement therapy and improvement in mammographic specificity. J Natl Cancer Inst 1997, 89:1623-1625.
9. Vachon CM, Sellers TA, Vierkant RA, Wu FF, Brandt KR: Case-control study of increased mammographic breast density response to hormone replacement therapy. Cancer Epidemiol Biomarkers Prev 2002, 11:1382-1388.

10. Litherland JC, Stallard S, Hole D, Cordiner C: The effect of hormone replacement therapy on the sensitivity of screening mammograms. Clin Radiol 1999, 54:285-288.

11. Banks E: Hormone replacement therapy and the sensitivity and specificity of breast cancer screening. A review. J Med Screen 2001, 8:29-34.

12. Marugg RC, van der Mooren MJ, Hendriks JH, Holland R, Ruijs $\mathrm{SH}$ : Mammographic changes in postmenopausal women on hormone replacement therapy. Eur Radiol 1997, 7:749-755.

13. Ozdemir A, Konus O, Nas T, Erbas G, Cosar S, Isik S: Mammographic and ultrasonographic study of changes in the breast related to HRT. Int J Gynecol Obstet 1999, 67:23-32.

14. Germer U, Tetzlaff A, Geipel A, Diedrich K, Gembruch U: Strong impact of estrogen environment on Doppler variables used for differentiation between benign and malignant breast lesions. Ultrasound Obstet Gynecol 2002, 19:380-385.

15. Nelson TR, Pretorius DH, Schiffer LM: Menstrual variation of normal breast NMR relaxation parameters. J Comput Assist Tomogr 1985, 9:875-879.

16. Martin B, El Yousef SJ: Transverse relaxation time values in MR imaging of normal breast during menstrual cycle. J Comput Assist Tomogr 1986, 10:924-927.

17. Rieber A, Nüssle K, Merckle E, Kreienberg R, Tomczak R, Brambs $\mathrm{HJ}$ : MR mammography. influence of menstrual cycle on the dynamic contrast enhancement of fibrocystic disease. Eur Radiol 1999, 9:1107-1112.

18. Müller-Schimpfle M, Ohmenhausen K, Stoll P, Dietz K, Claussen CD: Menstrual cycle and age. influence on parenchymal contrast medium enhancement in MR imaging of the breast. Radiology 1997, 203:145-149.

19. Heywang-Köbrunner SH, Viehweg $P$, Heinig A, Küchler C: Contrast-enhanced MR imaging of the breast. Accuracy, value, controversies, solutions. Eur J Radiol 1997, 24:94-108.

20. Hussain Z, Roberts N, Whitehouse GH, Garcia-Finana M, Percy $D$ : Estimation of breast volume and its variation during the menstrual cycle using MRI and stereology. Br J Radiol 1999, 72:236-245.

21. Reichenbach JR, Przetak C, Klinger G, Kaiser WA: Assessment of breast tissue changes on hormonal replacement therapy using MRI. A pilot study. J Comput Assist Tomogr 1999, 23: 407-413.

22. Kaiser WA, Zeitler E: MR-imaging of the breast: fast imaging sequences with and without Gd-DTPA. Radiology 1989, 170: 681-686.

23. Graham SJ, Stanchev PL, Lloyd-Smith CO, Bronskill MJ, Plewes $\mathrm{DB}$ : Changes in fibroglandular volume and water content of breast tissue during the menstrual cycle observed by MR imaging at 1.5 T. J Magn Reson Imaging 1995, 5:695-701.

24. Ottesen GL, Graversen HP, Blichert-Toft M, Zedeler K, Andersen JA: Lobular carcinoma in situ of the female breast. Am J Surg Pathol 1993, 17:14-21.

25. Neubauer H, Li M, Kuehne-Heid R, Schneider A, Kaiser WA: High grade and non-high grade ductal carcinoma in situ on dynamic MR mammography. characteristic findings for signal increase and morphological pattern of enhancement. $\mathrm{Br} J$ Radiol 2003, 76:3-12.

26. Taylor JS, Tofts PS, Port R, Evelhoch JL, Knopp M, Reddick WE, Runge VM, Mayr N: MR imaging of tumor microcirculation. promise for the new millennium. J Magn Reson Imaging 1999, 10:903-907.

27. Pike MC, Spicer DV, Dahmoush L, Press MF: Estrogens, progestogens, normal breast cell proliferation, and breast cancer risk. Epidemiol Rev 1993, 15:17-35.

28. Spicer DV, Ursin G, Parisky YR, Pearce JG, Shoupe D, Pike A, Pike MC: Changes in mammographic densities induced by a hormonal contraceptive designed to reduce breast cancer risk. J Natl Cancer Inst 1994, 86:431-436.

29. Fowler PA, Casey CE, Cameron GG, Foster MA, Knight $\mathrm{CH}$ : Cyclic changes in composition and volume of the breast during the menstrual cycle, measured by magnetic resonance imaging. Br J Obstet Gynecol 1990, 97:595-602.

30. Kuhl CK, Bieling HB, Gieseke J, Kreft BP, Sommer T, Lutterbey G, Schild $\mathrm{HH}$ : Healthy premenopausal breast parenchyma in 
dynamic contrast-enhanced MR imaging of the breast. Normal contrast medium enhancement and cyclical-phase dependency. Radiology 1997, 203:137-144.

31. Bogin L, Degani H: Hormonal regulation in orthotopic MCF7 human breast cancer. Cancer Res 2002, 62:1948-1951.

\section{Correspondence}

Dr Stefan OR Pfleiderer, Institute of Diagnostic and Interventional Radiology, Friedrich-Schiller-University Jena, Bachstr. 18, D-07740 Jena, Germany. Tel: +49 3641 933292; fax: +49 3641 934066; e-mail:stefan.pfleiderer@med.uni-jena.de 